\title{
Study on design of Linxia brick carving products based on shape grammar
}

\author{
Zhang Shutao*, Xu Juanjuan, Su Pengfei and Su Jianning \\ School of Design Art, Lanzhou University of Technology, Lanzhou, Gansu, 730050, China
}

\begin{abstract}
In view of the problems such as the single type of cultural derivative products of Linxia brick carving and the weak cultural attraction, we carried out the application research of Linxia brick carving culture by using shape grammar. Firstly, we collected and combed the materials of brick carving culture in Linxia by means of literature research and field investigation. Then, we determined the target product, analyzed the product form and emotional demand, construct the reference product form database, screen the brick carving pattern through the matching of the product emotional demand, obtained the characteristic lines of the brick carving pattern by the way of the type spectrum analysis, and established the typical form factor database of brick carving; Finally, taking some lines in the typical form factor database as the initial shape, we combined them with the lines in the reference product form database. According to the shape grammar deduction rules, the scheme was generated, the best scheme was selected and further refined. Studies have shown that the use of shape grammar for product design can enhance the cultural connotation of products, promote cultural heritage, and effectively guide the design of Linxia brick carving products.
\end{abstract}

\section{Introduction}

In recent years, with the implementation and promotion of national policies such as the revitalization of traditional crafts, poverty alleviation policy on intangible cultural heritage, and cultural and creative strategy, China's cultural and creative industry has become increasingly powerfull ${ }^{[1]}$, and has become an important pillar of national economic development. Cultural and creative products with traditional culture are widely favored by consumers. Culture injects inspiration into design, and design makes the spread of culture more dynamic. The effective use of traditional elements in the product design process can enhance the product competitiveness.

Linxia brick carving, as the first batch of intangible cultural heritage of the country, experiences a long period of development, and shows a continuous vitality. While the ancient brick carving art blooms the bright Chinese civilization, its traditional production way, material, application space, practitioner loss, and thinking solidification brought by "hand-by-hand" technology inheritance, hinder the development of the brick carving industry. The traditional creation subjects and expression forms do not conform to the aesthetic of new era, and have low adaptability to the current market environment, limit the promotion and application of products, and have a negative impact on its inheritance. Therefore, advancing with the times is an important challenge to the brick carving culture, and the transformation and upgrading of brick carving products are also urgent. Only by opening the way of thinking, bringing forth the new, and developing more popular products, can we promote the brick carving art to go further.

Based on this, from the perspective of product diversification and cultural heritage, this study introduces shape grammar to innovate the design of brick carving products.

\section{Shape grammar}

\subsection{Definition}

Shape grammar is a design method based on "shape" operation proposed by George Stiny and James Gips in the $1970 \mathrm{~s}^{[2]}$. It refers to the design reasoning method of generating a certain shape or more shape elements into a new shape in the spatial relationship according to the set rules ${ }^{[3]}$. According to the definition of shape grammar by George Stiny, shape grammar can be expressed as a formula: $S G=(S, L, R, I) . S G$ is the shape set derived by $S$ with certain regular orders such as rotation, mirroring, translation and other operation. $S$ is a finite set of shapes; $L$ is a finite set of tags; $R$ is a finite set with some deduction rules; $I$ is the initial shape ${ }^{[4]}$.

\subsection{Deduction rules}

The basic operation of shape grammar is to carry out the morphological evolution process of the initial form combined with the reference product family form 
according to certain rules ${ }^{[5]}$. Frequently-used shape grammar inference rules include replacement, scaling, addition and deletion, copying, rotation, mirroring, translation and fine-tuning ${ }^{[6]}$. Among them, replacement refers to replacing the morphological changes of target products with the morphological curves of different products. Addition and deletion refer to the operation of adding or deleting parts of the original initial form curve. Scaling refer to enlarge or shrink a part or whole of the initial shape curve. Copying means to place the original shape curve exactly from one position to another position, while the original shape unchanged. Rotation means to change the angle of the initial shape according to a certain axis or base point. Mirroring refers to the left and right (or front and back) symmetrical shape formed by turning part or all of the initial shape along an axis. Translation means to displacement of the initial shape. Fine-tuning refers to fine-tune the initial or evolved shape curve ${ }^{[7-8]}$.In the specific design application, there are a lot of intersections and repetitions of the shape inference rules ${ }^{[9]}$. For example, copying the shape can also be counted as an increase; while rotating the shape around the axis can be mirrored in some cases.

\subsection{Research status}

At present, shape grammar has been applied in quite a number of fields. Wang Yan, Wang Weiwei, Cui, etc. took the traditional patterns of various nationalities as research objects and applied shape grammar to the traditional pattern redesign. The results show that the new pattern design schemes are innovative and retain the genetic characteristics of traditional patterns ${ }^{[10-12]}$. Zhan Qinchuan applied shape grammar to the design of cultural and creative products, created a set of extraction process and method of solar term cultural characteristic factor, solved the problem of difficult extraction of characteristic factor contained in culture, and finally took the design of tea set as an example to verify the rationality of the method ${ }^{[13]}$. Qiao Xianling proposed the shape design method of Ru kiln porcelain ware based on shape grammar to form a new scheme. In combination with shape grammar ${ }^{[14]}$. Li Yang constructed a design model that satisfies cultural inheritance and morphological innovation to achieve the integration of peony culture and innovative design ${ }^{[15]}$.

These studies are all based on the shape grammar to study different objects. Through literature research, it is found that using shape grammar to extract cultural elements and apply them to the development of cultural and creative products is feasible. Therefore, we take brick carving culture as the research object on the basis of relevant literature materials, and propose a design and application method for brick carving cultural and creative products. Combining the extracted typical cultural elements of brick carvings with incense inserting seat products, the effectiveness of this method is verified. It is conducive to enhancing the cultural identity of the Linxia brick carving culture.

\section{$3 \quad$ Linxia brick carving}

Brick carving is a unique art form of architectural decoration in ancient Chinese architecture. It is made of green bricks and engraved with figures, flowers and plants, characters, landscapes and other patterns on surface ${ }^{[16]}$. It has a wide range of themes focusing on a better life, expressed by means of symbols, homophony, and harmonic meaning ${ }^{[17]}$, such as the yearning for more sons, more blessings and more longevity, the guidance and exhortation for humanistic ethics, and the willingness to succeed in career. And, its themes also contain some descriptions of the majestic mountains and rivers of the motherland, folk customs and other contents, which are rich in regional ethnic characteristics. There is few characters appear in Linxia brick carvings, which is also a distinguishing feature from other schools.

\section{Analysis}

\subsection{Analysis of target product}

In the minds of ancient Chinese literati, burning incense is an elegant thing. Burning incense, spraying tea, hanging pictures and arranging flowers are the "four arts of life" of Song dynasty literati ${ }^{[18]}$, which play a role in edifying sentiment, calming the mind and nourishing the nature, and also reflects the master's life attitude of pursuing freedom with nature. As a widely used incense burning tool, incense inserting seat is often integrated with incense to display the unique charm of incense culture. Through investigation, the existing design of incense inserting seat is diversiform, full of wit and interest. We often associate it with Zen culture to reflect people's need for spiritual realm improvement. The situation created by the shape of incense inserting seat enables us to immerse ourselves in a relaxed environment quickly through visual and emotional triggering. Therefore, the design of incense inserting seat should achieve the purpose of emptying mind and abandoning distractions.

\subsection{Construction of reference product form database}

The incense inserting seat is mainly composed of a base and a jack. According to the shape of the base, it can be divided into organic form, natural form and geometric form. In this paper, we collect the basic shapes of incense inserting seat form magazines, periodicals and networks to build product morphology database. The picture with side view can fully display the shape of the incense inserting seat. And the established reference product form database is designated as $I, I=\left\{I_{1}, I_{2}, I_{3} \ldots I_{\mathrm{n}}\right\}$, as shown in table 1 . 
Table 1. Reference product form database of incense inserting seat.

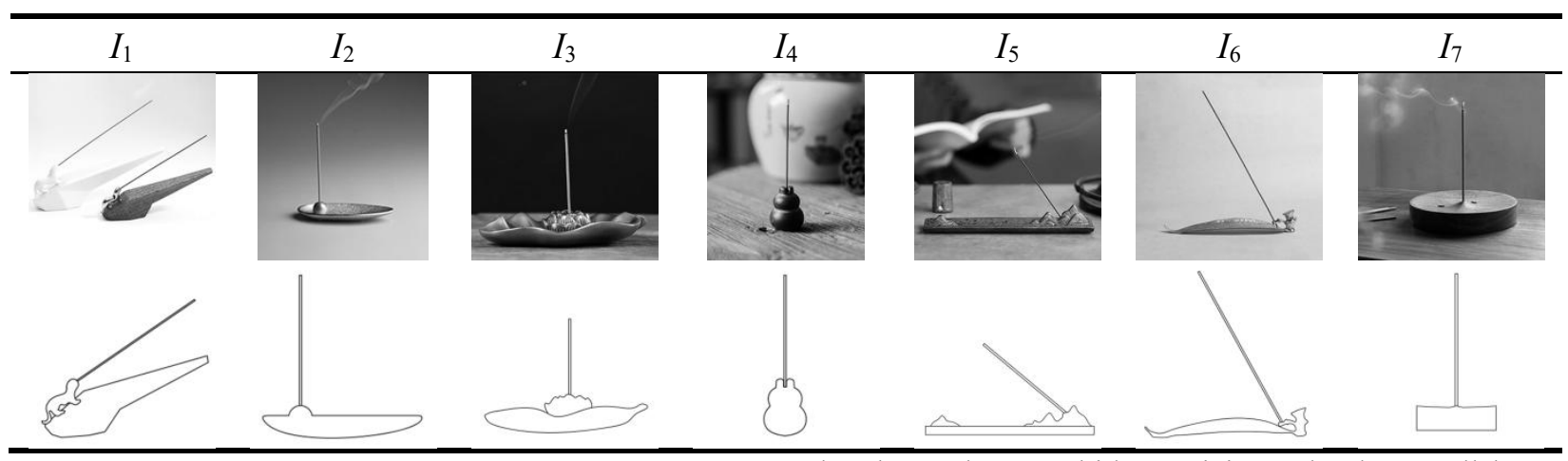

bamboo, plum, orchid, auspicious cloud, etc. all have a

\subsection{Pattern matching and screening}

Starting from the needs of Zen, elegance and sacredness embodied by incense inserting seat, we select patterns with matching semantics from the brick carving culture as the initial lines of the deduction of shape grammar form, as shown in table 2. The semantics of lotus, certain degree of agreement with the emotional needs of incense inserting seat. The lotus in the brick carving represents holiness, incorruptibility. And as a symbol of Buddha, it has a high matching degree with the spiritual needs of incense inserting seat in terms of Zen. So the lotus pattern is selected as the basic form.

Table 2. Semantic analysis.

\begin{tabular}{cccccc}
\hline $\begin{array}{c}\text { Plum } \\
\text { blossom }\end{array}$ & Orchid & Bamboo & Chrysanthemum & Lotus & $\begin{array}{c}\text { Auspicious } \\
\text { clouds }\end{array}$ \\
\hline $\begin{array}{c}\text { Noble, } \\
\text { Modest, } \\
\text { Strong }\end{array}$ & Noble, & $\begin{array}{c}\text { Noble, Humble, } \\
\text { Progant }\end{array}$ & $\begin{array}{c}\text { Faithful, Noble, } \\
\text { Auspicious, } \\
\text { Longevity }\end{array}$ & $\begin{array}{c}\text { Holy, } \\
\text { Buddhist symbol, } \\
\text { Incorruptible, Pure }\end{array}$ & $\begin{array}{c}\text { Auspicious, } \\
\text { Peaceful, Ideal, } \\
\text { Sacred }\end{array}$ \\
\hline
\end{tabular}

\subsection{Obtaining morphological factors of typical patterns based on type spectrum analysis}

Through investigation and network collection, the patterns of lotus brick carvings in Linxia East Mansion, Hongyuan, Bafang 13 alleys, Gongbei of Great Mosque and local brick carving factories were collected and sorted out. Type spectrum analysis is a way to classify samples with similar attributes according to certain rules
[19]. Based on the theory, we obtain the typical form factors of brick carving. Through the construction of feature atlas, brick carving samples are classified, and the significant characteristics are mined from a large number of samples. Because lotus and leaf usually coexist and are closely related, they should be extracted together. The two are sorted out and extracted to construct the characteristic set, as shown in table 3 and table 4 .

Table 3. Characteristic set of lotus leaf brick carving.

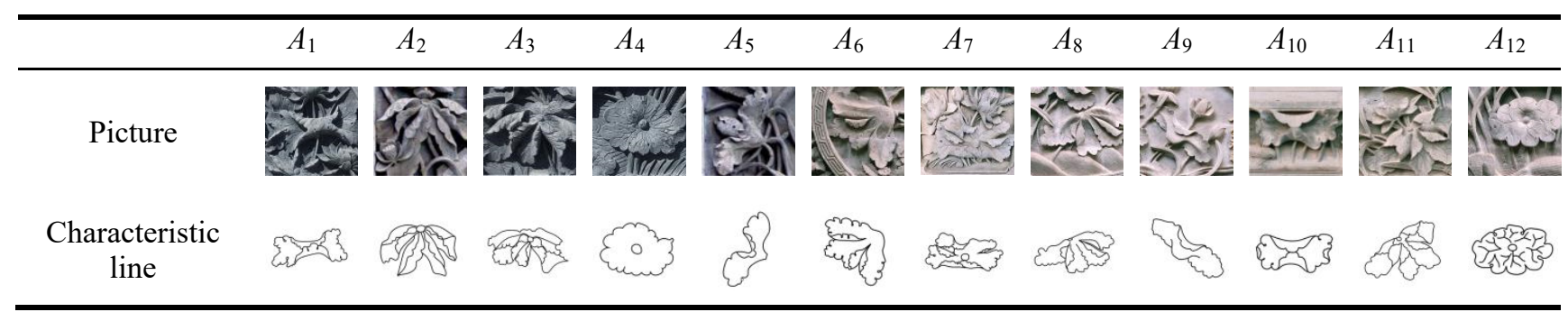

Table 4. Characteristic set of lotus brick carving.

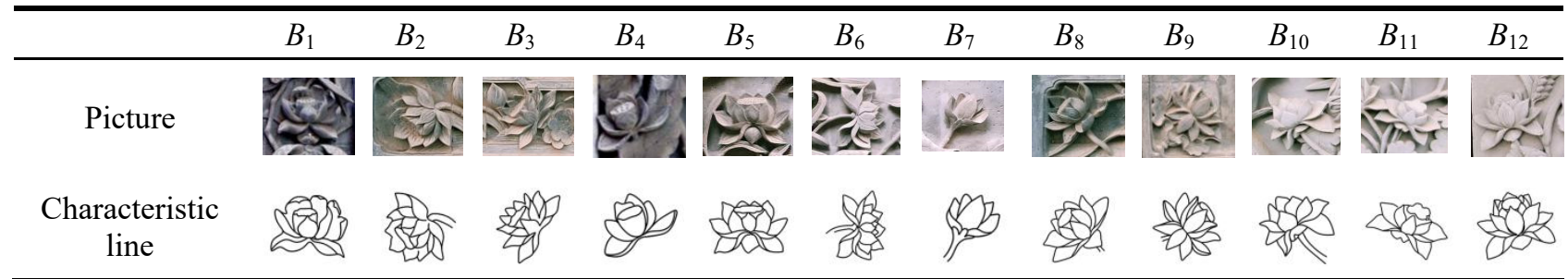

The characteristic curve set $A_{1}-A_{12}$ extracted from lotus leaf brick carving and $B_{1}-B_{12}$ extracted from lotus brick carving constitute the typical form factor database of brick carving. From a large number of Linxia brick carving graphic, we can find that the overall shape of the lotus leaf of Linxia brick carving is mostly in a curled state, and mostly rolled inwards from both sides. The 
edge of the rolled blade is composed of several arcs. The lotus is rich in layers and the petals are distinct. This can be used as a guide during the later form deduction to make the product more culturally inheritable and recognizable.

\section{Product innovation design based on shape grammar}

\subsection{Scheme generation and constraints}

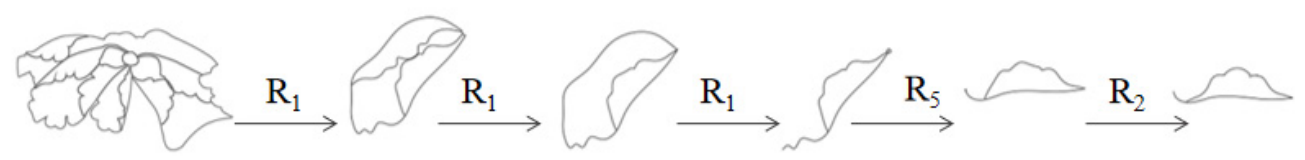

Figure 1. The deduction process of the initial line of the lotus leaf brick carving.
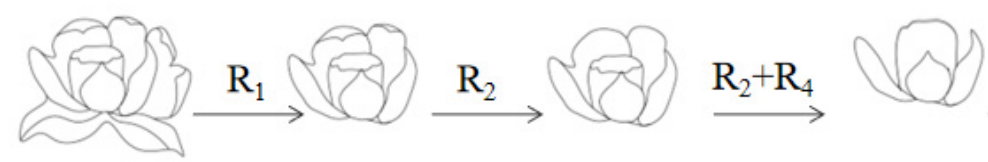

$\mathrm{R}_{2}$

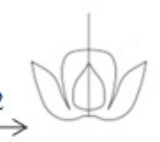

Figure 2. Deduction process of the initial line of the lotus brick carving.

We choose $I_{2}$ as the initial shape from the incense inserting seat reference product shape database, split the curves, carry out the initial line replacement operation on the base, socket and other parts of incense inserting seat. With the deduction rules, the shape of the incense inserting seat is deduced, and the deduction process of the incense inserting seat is shown in figure 3 . In the process of deduction, the grammar constraints are set as $10 \mathrm{~mm} \leq L$ (length) $\leq 240 \mathrm{~mm}, H$ (height $) \leq 50 \mathrm{~mm}$, and $10 \mathrm{~mm} \leq W$ (width) $\leq 80 \mathrm{~mm}$.

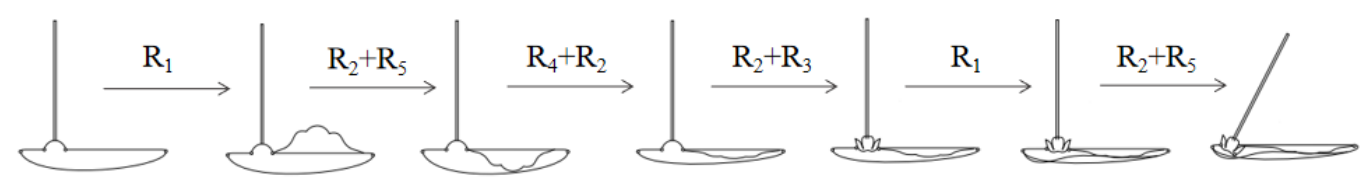

Figure 3. Deduction process of incense inserting seat shape.

\subsection{Scheme evaluation}

Applying shape grammar to carry out incense inserting seat design with cultural connotation of Linxia brick carving can generate a variety of schemes. Starting from the aspects of aesthetics, design sense and structure, the schemes are selected in the scheme group, 3-D modeling is carried out for the solution that meets the requirements. Combined with modern design concepts and formal beauty principles, the solution is optimized. The socket part of the incense inserting seat scheme is composed of the initial line replacement of the lotus brick carving, but it fails to reflect the characteristics of rich carving levels of lotus brick carvings in Linxia. Therefore, the shape of the socket part is optimized to make the adjusted product shape more in line with the needs of modern aesthetic concept, and the final scheme is output, as shown in figure 4 .

It can be seen that the final shape of the base part is curled and smooth; the socket part is exquisite and generous, the layers are distinct, and the whole is full of lingering charm. The word gives people an elegant and rustic feeling. The characteristics of Linxia brick carvings have been retained, the effect of inheriting the context is remarkable.

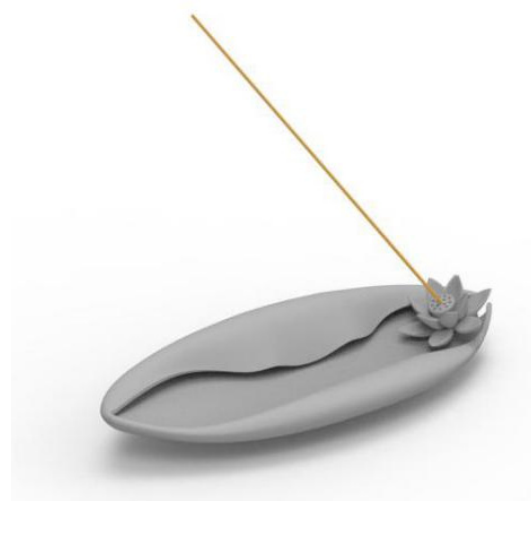

Figure 4. Final scheme.

\section{Conclusion}

Based on the shape grammar, we propose an innovative design method to derivative products of Linxia brick carving. Though the analysis of brick carving culture, we construct a cultural symbol extraction model which 
obtains the design factors of brick carving culture from the emotional needs of the target products, and represents the morphological genes by combining the type spectrum analysis. Combined with the reference product shape database, the shape grammatical inference rules are used to innovate the shape of incense inserting seat. Verified by an example, this method can effectively heritage context, enhance the attractiveness of cultural products, improve design efficiency.

\section{Acknowledgements}

The authors would like to acknowledge the financial support from the Science and Technology Department of Gansu Province (Grant No. 18CX4JN022), the National Natural Science Foundation of China (Grant No. 51705226), the Department of Education of Gansu Province (University Innovation Fund Project of Gansu Province, 2020, Research on innovative design method and theoretical system of cultural and tourism products).

\section{References}

1. Huang Zhihao. (2019) A Summary of Research on Cultural and Creative Product Design Based on Traditional Culture. Popular Literature and Art, (09): 97-98.

2. Stiny, G., Gips, J. (1972) Shape grammars and the generative specification of painting and sculpture. IFIP Congress North-Holland Publishing Company.

3. Stiny, G. (1980) Introduction to shape and shape grammars. Environment and Planning B: Planning and Design, 7(03): 343-351.

4. Lu Zhaolin, Tang Wencheng, et al. (2010) Discussion on Shape Grammar and Its Application in Industrial Design. Zhuangshi, (02): 102-103.

5. Yang Yanpu, Cheng Dengkai, Yu Suihuai, et al. (2013) Pan-ethnic group product form design based on shape grammar. Computer Integrated Manufacturing Systems, 19(9): 2107-2115.

6. Wang Weiwei, Yang Yanpu, Yang Xiaoyan, et al. (2014) Method of Product Form Design Based on Shape Grammar. Journal of Graphics, 35(1): 68-73.

7. Lu Zhaolin, Tang Wenchen, Xue Chengqi. (2010) Method of design DNA reasoning based on shape grammar. Journal of Southeast University (Natural Science Edition), 40(04): 704-711.

8. Luo Shijian, Zhu Shangshng, Feng Cheng (2008) Product Family Design DNA in Industrial Design. China Journal of Mechanical Engineering, 44(07): 123-128.

9. Stiny, G. (2006) Talking about Seeing and Doing. MIT Press, Cambridge.

10. Wang Yan, Cheng Kun (2020) Study on the translation and innovative design of Xinjiang Uyghur pattern. Industrial Design, (02): 133-135.

11. Wang Weiwei, Peng Xiaohong, Yang Xiaoyan. (2017) Application Study of Shape Grammar in
Evolutionary Design of Traditional Patterns. Packaging Engineering, 38(06): 57-61.

12. Cui J, Tang M X. (2013) Integrating shape grammars into a generative system for Zhuang ethnic embroidery design exploration. Computer Aided Design, 45(03): 591-604.

13. Zhan Qinchuan, Wang Ningxin, Li Ziyang, et al. (2019) Cultural and Creative Tea Set Design and Method Based on the Beginning of Spring. Packaging Engineering, 40(08): 176-181.

14. Qiao Xianling, Yu Xiaoqing, $\mathrm{Hu}$ Zhigang, et al. (2016) Method of Ruguan Kiln Porcelain Wares Form Design Based on Shape Grammar. China Ceramic, 52(11): 98-102+106.

15. Li Yang, Wang Wei. (2019) Innovative Design of Peony Cultural Product Form Based on Shape Grammar. Chinese Journal of Ergonomics, 25(04): 70-73.

16. Niu Le. (2011) Subi Qinghui-Research on Linxia Brick Carving Art. Tianjin Education Press, Tianjin.

17. Ge Haifei. (2017) Research on the Auspicious Pattern Design of Linxia Brick Carving. Art Science and Technology, 30(11): 181.

18. Shen Wenming. (2012) Display the historical charm of Chinese Incense Culture-2013 Shanghai Spring Agarwood Fair held in Shanghai. Shanghai Commercial, (04): 76-77.

19. Wang Ningxin. (2019) Visual Symbolization of Twenty-four Solar Cultures-Taking the Beginning of Spring as an example. Shaanxi University of Science and Technology. 